NBER WORKING PAPERS SERIES

\title{
HETEROGENEITY AND OUTPUT FLUCTUATIONS IN A DYNAMIC MENU-COST ECONOMY
}

\author{
Ricardo J. Caballero \\ Eduardo M.R.A. Engel
}

Working Paper No. 3729

\author{
NATIONAL BUREAU OF ECONOMIC RESEARCH \\ 1050 Massachusetts Avenue \\ Cambridge, MA 02138 \\ June 1991
}

This paper is part of NBER's research program in Economic Fluctuations. Any opinions expressed are those of the authors and not those of the National Bureau of Economic Research. 
NBER Working Paper \#3729

June 1991

\section{HETEROGENEITY AND OUTPUT FLUCTUATIONS \\ IN A DYNAMIC MENU-COST ECONOMY}

\section{ABSTRACT}

When firms face menu costs, the relation between their output and money is highly nonlinear. At the aggregate level, however, this needs not be so. In this paper we study the dynamic behavior of a general equilibrium menu-cost economy where firms are heterogeneous in the shocks they perceive, and the demands and adjustment costs they face. In this context we (i) generalize the Caplin and Spulber [1987] steady state monetary neutrality result; (ii) show that uniqueness of equilibria depends not only on the degree of strategic complementarities but also on the degree of dispersion of firms' positions in their price-cycle; (iii) characterize the path of output outside the steady state and show that as strategic complementarities become more important, expansions become longer and smoother than contractions; and (iv) show that the potential effectiveness of monetary policy is an increasing function of the distance of the economy from its steady state, but that an uninformed policy maker will have no effect on output on average.

Ricardo J. Caballero Department of Economics Columbia University New York, NY 10027 and
Eduardo M.R.A. Engel Department of Economics M.I.T.

Cambridge, MA 02139

NBER 


\section{INTRODUCTION}

An important part of the debate on the response of prices to monetary shocks has centered on the dynamic discrepancy between economies with and without price rigidities, on the magnitude and persistence of these discrepancies, and on whether the monetary authority should attempt to exploit rigidities or not.

These issues have been typically addressed in a framework in which individual prices (or wages) adjust according to some fixed time-schedule, resulting in a sluggish aggregate price level. Caplin and Spulber [1987] (henceforth CS) show that such an assumption is not as innocuous as may seem - when the fixed time-schedule approach is abandoned in favor of having individual prices adjust according to a rule limiting the size of the departure from a target real price (a state dependent rule), results on monetary policy effectiveness may change dramatically. They provide an example where individual prices are controlled infrequently according to simple state dependent rules, but where the aggregate price level is fully flexible respect to certain types of monetary shocks (both anticipated and unanticipated).

CS's economy, while dynamic, starts off and remains at its steady state; the assumptions they make ensure that - except for a location parameter determined by the current level of the money stock - the distribution of prices is self-replicating. Many new issues arise when the shape of this distribution is allowed to change over time. For example, does the economy have any natural forces pushing it towards the CS limit? What is the path of output while outside the steady state? Is there a unique equilibrium path? Which is the role played by strategic interactions in shaping the dynamic path of output? Is there a role for monetary policy?

This paper provides a framework within which some of these questions can be answered. We consider the simple microeconomic state dependent rule used by CS; the fixed $(S, s)$ rule, which can be justified by the presence of fixed costs of adjusting prices ("menu costs"). This pricing rule is optimal in certain sections of the paper; in others it corresponds to an approximation of the first best rule. Under the maintained assumption of fixed $(S, s)$ pricing rules, we describe the endogenous evolution of the distribution of prices, and the way this affects both output fluctuations and the response of output to monetary shocks.

The basic macroeconomic framework - which corresponds to an extension of Blanchard and Kiyotaki [1987] to a dynamic setting- is presented in Section 2, Section 3 extends the 
CS neutrality result to the case where firms differ in the shocks they are subject to, the adjustment costs they perceive, and the demand elasticities they face.

In Section 4 we show that whether uniqueness can be guaranteed or not depends not only on the degree of strategic complementarity, as is the case when only symmetric equilibria are considered (e.g. Cooper and John [1988]), but also on the degree of dispersion of firms' price deviations; the more dispersed these are, the stronger the degree of strategic complementarities necessary to yield multiplicity.

Section 5 characterizes the path of output when the economy is outside its steady state. We show that, in the presence of positive core inflation, strategic complementarities introduce realistic asymmetries into the business cycle generated by the model; the stronger these complementarities, the longer and smoother expansions are relative to contractions.

Section 6 shows that monetary policy that uses information on the level of output and firms' prices has effects on output outside the steady state. Yet monetary policy remains neutral on avenag; the average effect of monetary policy when no information about the location of the distribution of firms' price deviations is available is equal to zero. Section 7 presents concluding remarks; it is followed by several appendices.

\section{MaCROECONOMIC FRAMEWORK}

Mankiw [1985] shows the potential first order effects of monetary policy when small (second order) non-convex costs of adjusting prices are present and competition is imperfect. ${ }^{1}$ Recent static general equilibrium models have furthered our understanding of the macroeconomic role of such costs (e.g. Blanchard and Kiyotaki [1987], Rotemberg [1987]). These models have several elements in common: (a) an aggregate output equation relating output to real balances (aggregate demand) at a given instant in time, $Y(t)=G(M(t) / Q(t))$, with $Y, M$ and $Q$ denoting aggregate output, money stock and aggregate price level, respectively, and $G^{\prime}>0$; (b) a frictionless pricing equation for each firm $q_{i}^{*}(t)=H(Q(t), M(t) / Q(t))$, with $q_{i}^{*}$ denoting the $i$-th firm's optimal (private) frictionless price, and $H_{1}>0, H_{2}>0$; (c) a menu cost of changing prices so that the actual price charged by firm $i, q_{i}$, may differ from its optimal frictionless price within some range; (d) a symmetric aggregate price index; (e) the assumption that prices are at their frictionless optimal level before the monetary shock; (f) the assumption that equilibria are symmetric and (g) the assumption that cost

\footnotetext{
${ }^{1}$ Akerlof and Yellen [1985] give a similar insight in terms of near rationality.
} 
and demand structures are the same across firms.

If prices can be changed costlessly, then $q_{i}(t)=q_{i}^{*}(t)=Q(t)$, and money is neutral. An increase in the money stock is offset one for one by an equivalent increase in the price level. However, in the presence of menu costs there is a range in which actual prices do not increase with the money stock, although frictionless prices do. As a result, the aggregate price level does not match the increase in the money stock. This raises real balances and, through aggregate demand, output.

Caplin and Spulber [1987] extend the previous model to a dynamic setting. They use results on optimal dynamic pricing rules in the presence of fixed costs of price adjustments (Barro [1972], Sheshinski and Weiss [1977] and [1983]) to give more structure to assumption (c). Under certain conditions on the process describing the path of money, including monotonicity, the presence of menu costs leads firms to follow one sided, fixed, $(S, s)$ pricing rules. The price charged by a firm remains fixed until enough inflation (possibly not realized) accumulates so that its price is a given fraction $s$ below its frictionless optimal value. Once this trigger level is reached, firms reset their price to a fraction $S$ above their frictionless optimal price. Formally, denoting $\log q_{i}$ and $\log q_{i}^{*}$ by $p_{i}$ and $p_{i}^{*}$, respectively, the above pricing rule implies that $p_{i}(t)-p_{i}^{*}(t)$ belongs to $(s, S]$ for all $i$ and $t$. A continuum of firms is considered: $i \in[0,1]$, and the difference between the logs of the $i$-th firm's actual and frictionless optimal price is denoted by $z_{i}(t)$, which therefore belongs to $(s, S]$. In order to remove constants that are irrelevant in our analysis, we assume that $s=-S$.

CS also dispose of assumption (e). The reason for doing this is that in a menu cost economy where monetary shocks occur more than once firms are not at the point where $z_{i}(t)=0$ before every shock. Violation of condition (e) is important since the effects of monetary policy become ambiguous. For example, if all firms are bunched close to their trigger point $s$, a (positive) monetary shock is likely to lower real balances and output instead of raising them.

If the money stock increases continuously, the logarithm of a firm's nominal price increases by a quantity equal to the width of the corresponding $(S, s)$ band every time a firm adjusts its price; this amount is denoted by $\lambda$. Substituting the expression for $q_{i}^{*}$ shown in (b) in the definition of $z_{i}(t)$, yields $z_{i}(t)$ as a periodic function - with period $\lambda$ - of: $(i)$ the initial conditions faced by the $i$-th firm, $z_{i}(0)$, (ii) the logarithm of the aggregate price 
level, $P(t)$, and (iii) the logarithm of the aggregate money stock, $m(t)$ :

$$
z_{i}(t)=S-F\left(z_{i}(0), P(t), m(t)-P(t)\right)(\bmod \lambda)
$$

where $x(\bmod \lambda)$ denotes the remainder of dividing $x$ by $\lambda$.

Suppose initially $F\left(z_{i}(0), P(t), m(t)-P(t)\right)(\bmod \lambda)=0$. That is, the $i$-th firm just changed its price and therefore is at the target level $S$. If now $m(t)$ rises, ceteris paribus, real balances rise. This puts upward pressure on the firm's frictionless optimal price and lowers $z_{i}(t)$. This continues happening as the money stock rises bringing $F(\cdot, \cdot, \cdot)$ closer to $\lambda$, thus $z_{i}(t)$ closer to the trigger level $s$. Once $z_{i}(t)$ reaches $s$, the price is immediately reset to $S$, starting a new cycle.

The effects of changes in $P(t)$, also ceteris paribus, are less clear since substitution and real balances effects play opposing roles on the determination of the frictionless optimal price. An increase in $P(t)$ raises $p_{i}^{*}(t)$ through the substitution effect but lowers it through the real balances effect. This tradeoff is a well known source of multiple equilibria (e.g. Ball and Romer [1987]) and affects the dynamic response of output to monetary shocks in important ways; these issues are discussed in Sections 4 and 5.

CS showed that money is neutral when the above assumptions are combined with an initial distribution of (the logarithm of) prices that is uniform on $(s, S]$. Here we drop the symmetry assumptions made in CS and the models mentioned above. This allows us to address interesting non-steady state issues and study the generality of CS's steady state neutrality result. This is done in four ways: (a) ther can be an arbitrary distribution of firms' initial positions within their pricing cycle; (b) there are firm-specific cost and demand shocks (idiosyncratic shocks), (c) the cost of changing prices may differ across firms, and (d) there may be differences in demand elasticities across firms. Extension (b) corresponds to modifying the optimal pricing equation so that $q_{i}^{*}(t)=H\left(Q(t), M(t) / Q(t), W_{i}(t)\right)$, where $W_{i}(t)$ represents shocks affecting only firm $i$ (and $w_{i}(t)$ its logarithm), with (just for convention) $H_{3}>0$. Equation (1) then becomes:

$$
z_{i}(t)=S_{i}-F\left(z_{i}(0), P(t), m(t)-P(t), w_{i}(t)\right)\left(\bmod \lambda_{i}\right)
$$

with $s_{i}$ and $S_{i}$ denoting firm specific trigger and target points and $\lambda_{i}$ their difference (i.e. $\lambda_{i}=S_{i}-s_{i}$ ). Thus positive idiosyncratic shocks lower $z_{i}(t)$ (whenever the trigger level $s_{i}$ is not reached) by raising the $i$-th firm's optimal frictionless price. An extension of 
Blanchard and Kiyotaki's [1987] (henceforth BK) model, presented in the appendix, yields the following functional form for $z_{i}(t)$ :

$$
z_{i}(t)=S_{i}-\left(S_{i}+m(t)+w_{i}(t)+k \int_{0}^{1} z_{u}(t) d u-p_{i}(0)\right)\left(\bmod \lambda_{i}\right) .
$$

If $m(t)$ and $w_{i}(t)$ are interpreted as deviations from their values at time $t=0$, and $\Delta z_{i}(t) \equiv$ $z_{i}(t)-z_{i}(0)$, then equation (3) is equivalent to:

$$
z_{i}(t)=S_{i}-\left(S_{i}+m(t)+w_{i}(t)+k \int_{0}^{1} \Delta z_{u}(t) d u-z_{i}(0)\right)\left(\bmod \lambda_{i}\right)
$$

The term $k \int z_{u} d u$ in (3) reflects the fact that firms look at the aggregate price level (i.e. real balances and substitution effects do not necessarily cancel) when setting their prices. Consider the case where $k$ is positive and the money stock is fixed. If firms' prices are (on average) above their frictionless optimu $m$, the $i$-th firm has more pressure (on average) to raise its price. Conversely, if on average other firms' prices are below their frictionless optimal price then the $i$-th firm has less pressure to change its price. This corresponds to the concept of strategic complementarity. If $k$ is negative, there is strategic substitutability between firms.

The model underlying equation (3) can be extended to incorporate heterogeneity in the relation between firms' (or sectors') behavior and the business cycle. We introduce this realistic feature by letting the income elasticities of the demands faced by firms differ. ${ }^{2}$ This leads to an expression analogous to (3) with $k+1-\beta_{i}$ in the place of $k$, where $\beta_{i}$ is the income elasticity of the demand faced by firm $i$ and $\int \beta_{i} d i=1$. Firms with large values of $\beta_{i}$ have more incentives to raise their prices when output is above average than firms with small values of $\beta_{i}$.

Defining the (logarithm of the) price level by $P(t)=\int_{0}^{1} p_{i}(t) d i$, using the definition of $z_{i}(t)$ and the fact that in this model $y(t) \equiv \log Y(t)=m(t)-P(t)$, it follows that output is a linear function of the average deviation of prices from their frictionless optima:

$$
y(t)=-(1+k) \int_{0}^{1} z_{i}(t) d i
$$

Output falls (rises) when the average price deviation (from the frictionless optima) rises (falls). Any effect of money on output comes through its effect on this average.

\footnotetext{
${ }^{2}$ Allowing for heterogeneity in price elasticities is a trivial extension of the case we consider.
} 


\section{Principle of Uniformity and Neutrality}

In this section we show that the principle underlying Caplin and Spulber's neutrality result remains valid in the presence of various sources of heterogeneity among firms. Underlying this result is the fact that once the distribution of firms within their pricing cycle is uniform, it remains uniform as long as the sources affecting firms' prices are independent from their price deviations.

In order to make the above statements precise, we begin by defining the cross-section distribution of firms' price deviations at time $t, F_{t}(z)$. Other cross-section distributions are defined analogously. The quantity $F_{t}(z)$ is equal to the fraction of firms with price deviations less than or equal to $z .^{3}$ It describes the distribution of firms' percentage deviations that actually attains at time $t$, and is totally unrelated to the observer's state of knowledge. We refer to it as the "distribution of price deviations," for short.

Next we derive the steady state distribution in the simplest case, where firms have the same $(S, s)$ bands and demand elasticity parameters, and no strategic interactions are present. Let $z_{t}$ and $w_{t}$ denote random variables whose (joint) distribution function coincides with the (joint) cross-section distributions of the $z_{i}(t)$ 's and $w_{i}(t)$ 's, respectively. Thus, for example, $z_{0}$ denotes the initial distribution of price deviations. Equation (4) then leads to:

$$
z_{t}=S-\left(S+m(t)+w_{t}-z_{0}\right)(\bmod \lambda)
$$

If no idiosyncratic shocks are present $\left(w_{t} \equiv 0\right.$ for all $\left.t\right)$ and $z_{0}$ is uniformly distributed on the interval $(s, S)$, then the distribution of price deviations, $z_{t}$, is also uniform. The level of $m(t)$ determines the relative position of firms within their cycle and the number of times they have changed their prices, but it does not affect the shape of the distribution of price deviations. CS showed that real balances and hence output cannot be affected by monetary policy in this framework. A continuous increase of the money stock by $\Delta m$ leads a fraction $\Delta m / \lambda$ of firms to increase their prices by $\lambda$. Thus the product of the fraction of firms changing their prices and the size of these changes - the change in the aggregate price index- is $\Delta m$, leaving real balances (hence activity) unchanged.

Idiosyncratic shocks have no impact on the relation between money and output once the economy is at its steady state. Monetary neutrality follows from the fact that if the initial

\footnotetext{
${ }^{3}$ This distribution function is rigorously defined when the number of firms is finile. Considering a continuum of firms should be interpreted as a notationally convenient way of dealing with a large but finite nu mber of firms.
} 
distribution of price deviations is uniform on $(s, S]$, then the distribution of prices at time $t$, $z_{t}$, is also uniform on $(s, S]$. This holds regardless of the distribution generating idiosyncratic shocks, as long as its increments are independent from current prices. Intuitively, if prices start off at their steady state and there is nothing systematic in the way idiosyncratic shocks occur, then all these shocks effectively do is change the relative order of firms within the $(S, s)$ interval, without affecting the fraction of firms with price deviations within any particular interval.

Next we allow the width of the $(S, s)$ bands to differ across firms. Now some firms have high costs of adjusting their price and therefore allow their price deviation to vary considerably while others have small adjustment costs and change their prices more often. The steady state distribution of price deviations (the $z_{i}(t)$ 's) is no longer uniform. The variable that is uniformly distributed is the fraction every firm has covered of its own pricing cycle at a given instant in time. Formally, let $c_{i}(t) \equiv\left(S_{i}-z_{i}(t)\right) / \lambda_{i}$ denote the fraction of its current cycle covered by the $i$-th firm at time $t$, and $c_{t}$ denote the corresponding crosssection distribution. This variable takes values between zero and one. From equation (6) it follows that

$$
c_{\ell}=\left(c_{0}+\frac{m(t)+w_{t}}{\Lambda}\right)(\bmod 1)
$$

where $\Lambda$ denotes a random variable with the same cross-section distribution as the $\lambda_{i}$ 's. If the initial position of firms within their cycle $\left(c_{i}(0)=\left(S_{i}-z_{i}(0)\right) / \lambda_{i}\right)$ is uniform, then it remains uniform under rather weak conditions. All that is needed is that the (joint) crosssection distribution of bandwidths and increments of idiosyncratic shocks be independent from firms' current positions within their pricing cycle, i.e. that $\left(d w_{t}, \Lambda\right)$ be independent from $c_{\ell}$. The proof is similar to the one we sketched above. Neutrality of money is derived by noting that equations (5) and (7) imply that

$$
y(t)=(1+k)\left(\int_{0}^{1} \lambda_{i} c_{i}(t) d i-S\right)
$$

with $S=\int S_{i} d i$. The integral (expectation) on the right hand side of (8) is calculated by first conditioning on the value of $\lambda_{i}$, then applying the result for the case of equal $(S, s)$ rules ${ }^{4}$ and finally adding up over all possible values of $\lambda_{i}$. This shows that in the steady state $y(t)=\frac{1}{2} \int \lambda_{i} d i-S=0$. Thus $y(t)$ is unaffected by money changes. Firms increase

\footnotetext{
'The assumption that $c_{t}$ is independent from $\left(d w_{t}, \Lambda\right)$ is used at this step.
} 
their prices by amounts proportional to their bandwidths, yet this is offset by the fact that the proportion of firms changing their prices within each group (defined as firms with the same $\lambda$ 's) is inversely proportional to the width of firms' inaction bands.

Adding strategic interactions and heterogeneity in elasticities does not affect the steady state nature of the uniform distribution of $c_{t}$, since these only play a role outside the steady state, when output fluctuates (see equation (3) and Section 5). The main result of this section is summarized in the following proposition.

Proposition 1 (Principle of Uniformity): Assume the cross-section distribution of firms' initial positions within their (pricing) cycle, $c_{0}$, is (a) uniform on $[0,1)$ and (b) independent of the (joint) cross-section distributions of idiosyncratic shocks (that take place at time $t>0$ ) and bandwidths. Then (a) the cross-section distribution of firms' positions within their cycle at time $t, c_{t}$, also is uniform on $[0,1)$ and (b) monetary policy (that increases continuously and monotonically, and does not affect firms' pricing rules) is neutral.

Proof Any solution to the simultaneous set of equations defined by (3) at time $t$ defines a cross-section distribution of firms positions within their pricing cycle of the form (see Section 4):

$$
c_{t}=\left(c_{0}+\frac{m(t)+w_{t}+(k+1-\beta) r}{\Lambda}\right)(\bmod 1)
$$

where $\beta$ denotes a random variable with the same distribution as the cross-section distribution of the $\beta_{i}$ 's, and $r$ is a constant (that depends on $\beta, t, k, \Lambda$ and $m(t)$ ) equal to zero in steady state.

Equation (9) shows that the cross-section distribution of firms within their pricing cycle at time $t$ is the sum, mod 1 , of a distribution uniform on $[0,1)$ and a distribution independent from the latter. That the resulting distribution again is uniform on $[0,1)$ under these assumptions follows from standard Fourier analysis and is shown in Lemma B1 in Appendix B. The conditioning argument given in the text can then be used to show that output remains constant over time.

Thus, CS's steady state result can be extended to the case where idiosyncratic shocks, different $(S, s)$ rules and different demand elasticities are present. The aggregate behavior of the corresponding economy -including monetary neutrality - is indistinguishable from that of an economy with equal $(S, s)$ bands and demand elasticities across firms, and without idiosyncratic shocks. Yet at the microeconomic level there is an additional element of 
realism in the model presented above. The relative position of two firms within their cycle may change over time due to idiosyncratic shocks or aggregate shocks (or both). The latter only occurs if the firms' $(S, s)$ bands have different widths since it is only in this case that aggregate shocks displace firms by different fractions of their cycles. Also the empirical distribution of prices need not be uniform since $p_{t}=z_{t}+p_{t}^{*}$, and the distribution of $p_{t}^{*}$ is likely to be dominated by the distribution of idiosyncratic shocks. Furthermore, if the $(S, s)$ bands are different across firms, $z_{t}$ is not uniform. ${ }^{5}$

\section{Existence, Uniqueness and Multiple Equilibria}

When income and substitution effects cancel $(k=0)$, equation (3) defines the (unique) equilibrium of the $(S, s)$ economy. This section highlights some of the issues involved in determining existence and uniqueness of an equilibrium when this is not the case. Firms' deviations from their frictionless prices, the $z_{i}(t)$ 's, then appear on both sides of $(3)$ and it is not obvious that this system of equations has a solution. We first show that an equilibrium exists under very weak assumptions (Section 4.1). This is followed by the derivation of conditions that ensure uniqueness (Section 4.2). These conditions require that either the degree of strategic complementarity across firms be small or that the economy be close to its steady state. The section concludes with some speculations on what happens when the possibility of more than one equilibrium arises. The results we obtain should be viewed as a first step towards understanding equilibria in dynamic menu costs economies with strategic interactions, since the fixed $(S, s)$ rules assumption is (more) likely to be suboptimal in this case.

In order to highlight the interrelation between the degree of strategic complementarity and the existence and uniqueness of equilibria, we assume bandwidths and demand elasticities are the same across firms. This section's results can be extended to the general macroeconomic framework considered in Section 2, as was done in a working paper version

\footnotetext{
${ }^{3}$ This does not include the stochastic bands case considered in Benabou [1989]. However the principles underlying the proots in this paper are likely to extend to his setting.
} 
of this paper (Caballero and Engel [1989b]).

\subsection{Existence and uniqueness}

Consider again the economy described in Section 2 and summarized by the equation

$$
z_{i}(t)=S-\left(S+m(t)+w_{i}(t)+k \int_{0}^{1} z_{u} d u-p_{i}(0)\right)(\bmod \lambda)
$$

A collection $\left\{p_{i}(t) ; i \in[0,1]\right\}$ is said to define a general equilibrium at time $t$, if the corresponding $z_{i}(t)$ 's - which are uniquely determined since the frictionless economy always has a unique equilibrium (see Appendix A)-- solve the system of simultaneous equations defined by (10). Thus determinig whether an equilibrium exists at time $t$ is equivalent to determining whether there exists a collection $\left\{z_{i}(t) ; i \in[0,1]\right\}$ that solves $(10)$.

If $\left\{z_{i}(t) ; i \in[0,1]\right\}$ defines a solution of $(10)$, then there exists a real number $r(t)$ (equal to $\left.\int z_{i}(t) d i\right)$ such that

$$
z_{i}(t)=S-\left(S+m(t)+w_{i}(t)+k r(t)-p_{i}(0)\right)(\bmod \lambda) .
$$

Hence any two solutions of (10) differ only in the value of $r(t)$ in (11). Thus if we compare the cross-section densities of firms' positions within their price cycle associated with two different equilibria, any one of them is a mod-1 rotation of the other. ${ }^{6}$

Comparing equations (10) and (11) shows that proving existence of a solution at time $t$ is equivalent to finding a number $r$-that typically depends on the instant of time $t$ being considered- such that

$$
r=\int_{0}^{1} z_{u}(t) d u
$$

where the $z_{i}(t)$ 's are given by (11), as a function of $r$. Let $Y_{t}$ denote a random variable with a distribution equal to the cross-section distribution of the $\left(S+m(t)+w_{i}(t)-p_{i}(0)\right)$ 's. From (11) it follows that solving (12) is equivalent to solving the following (fixed point) equation:

$$
S-r=\mathrm{E}\left(Y_{t}+k r\right)(\bmod \lambda)
$$

The left and right hand sides of (13) are denoted by $L(r)$ and $G_{t}(r)$, respectively. The function $G_{t}(r)$ is periodic (with period $\lambda /|k|$ ). We denote its maximum and minimum

\footnotetext{
${ }^{6}$ The random variable $Y$ is a $\bmod -1$ rotation of $X$ if $Y(X+a)(\bmod 1)$ for some constant $a$.
} 
values by $G_{\max }$ and $G_{\min }$, respectively. Equation (13) then shows that we may restrict our attention to values of $r$ in $\left[S-G_{\max }, S-G_{\min }\right]$. Both functions take values between $m$ and $M$ on this set. We therefore have that the existence of an equilibrium is equivalent to having a curve restricted to a square (with side of length $G_{\max }-G_{\min }$ ) intersect the second diagonals of that square. A sufficient condition for existence of an equilibrium is therefore that $G_{t}(r)$ be continuous (in $r$ ). This is the case, for example, if the initial distribution of prices and the distribution of idiosyncratic shocks are independent and any one of them has a density. ${ }^{7}$ This provides a simple characterization of any equilibrium of an economy described by a system of equations like (10).

A calculation from first principles shows that under the symmetric equilibria assumption (which implies that $Y_{t}$ is concentrated at one point and therefore does not have a density) the functions $L(r)$ and $G_{t}(r)$ do not necessarily cross. There often does not exist a solution for (10) when this is the case. At the opposite extreme, when the economy is at its steady state and $Y_{t}(\bmod \lambda)$ is therefore uniform, the constant $r$ solving the fixed point equation is unique and equal to zero, since output does not fuctuate $\left(G_{\max }=G_{\min }=0\right)$. This suggests that uniqueness depends on how near the cross-section distribution of firms' positions within their cycle is from the steady state.

Having established that an equilibrium exists (at time $t$ ) if $G_{t}(r)$ is continuous, additional conditions ensuring uniqueness are now considered. If two values of $r$ solve (13), then necessarily the slope of $G_{t}(r)$ (as a function of $r$ ) has to be equal the slope of the diagonal at some intermediate point (this is just a statement of the Mean Value Theorem). Hence $G_{t}^{\prime}(r)$ is equal to -1 for some $r$. As $G_{t}(r)$ is periodic, its derivative is equal to zero at some point. Therefore there exists a unique equilibrium when $G^{\prime}{ }_{t}(r)$ is continuous and $G^{\prime}{ }_{t}(r)>-1$ for all $r$. This is equivalent to having $k\left(1-h_{t}(1-k r)\right)>-1$ for all $r$ in $\left[G_{\min }, G_{\max }\right]$, where $h_{t}(z)$ denotes the density of $Y_{t}{ }^{8}$ Since the range of admissible values for $k$ is $(-1,+\infty),{ }^{9}$ it follows that there exists a unique solution whenever there is strategic substitutability (or no strategic interaction at all). The possibility of more than one equilibrium arises only when there exists strategic complementarity between firms' pricing decisions. The

\footnotetext{
${ }^{7}$ The proof follows from the fact that if the distribution of $Y$ has a density (with respect to Lebesgue measure) then the continuous mapping theorem (see Billingsley [1986, p.344]) implies that $G_{1}(r)$ is continuous. The conditions mentioned above ensure that $Y$ has a density.

${ }^{B} G_{i}^{\prime}(r)$ follows from Lemma B4 in Appendix B.

'The extension of Blanchard and Kiyotaki's model presented in the appendix requires short run decreasing returns to scale in ofder to have a bounded equilibrium. This restriction determines that $k>-1$ (see the appendix).
} 
condition ensuring uniqueness derived above holds when $\sup _{z}\left|h_{t}(z)-1\right|<1 / k$, which (as shown in the appendix) is equivalent to $\mathrm{d}_{R}\left(z_{\ell}, U\right)<1 / k$, where $\mathrm{d}_{R}\left(z_{\ell}, U\right)$ denotes the largest relative error made in approximating the distribution of price deviations by its steady state distribution. ${ }^{1011}$ The larger the degree of strategic complementarity, the closer the distribution of price deviations must be to its steady state to ensure uniqueness.

The results on existence and uniqueness of equilibria are summarized in the following proposition.

\section{Proposition 2 (Existence, Uniqueness and Continuity of Equilibria)}

Let $Z_{t}$ denote a random variable with the same distribution as the cross-section distribution of the $\left(w_{i}(t)-p_{i}(0)\right)$ 's.

Existence: Assume that $Z_{t}$ has a density (with respect to Lebesgue measure). Then the set of simultaneous equations defined by (10) has a solution (at time t). Further, all solutions are such that the cross-section distribution of firms' positions within their pricing cycle is of the form:

$$
c_{t}=\left(c_{0}+\frac{m(t)+w_{t}+k r(t)}{\lambda}\right)(\bmod 1)
$$

for some real number $r(t)$.

Uniqueness: Assume $H(a)=\mathrm{E}\left(Z_{t}+a\right)(\bmod \lambda)$ is continuously differentiable. ${ }^{12}$ Let $c_{t}$ denote a random variable that has the same distribution as the cross-section distribution of firms' positions with in their price-cycle associated with a particular equilibrium at time t. Then a sufficient condition for uniqueness is that either $k \leq 0$ or $\mathrm{d}_{R}\left(c_{t}, U\right)<1 / k$, whene $\mathrm{d}_{R}\left(c_{t}, U\right)$ denotes the largest relative error made when approximating the probability of events under $c_{t}$ by the probability assigned to that event by the steady state distribution.

Further, (the unique value of) output at time $t$ is the only solution of the following fixed point problem:

$$
y(t)=\frac{\lambda}{\phi}\left\{\mathrm{E}\left(c_{0}+\frac{m(t)+w_{t}-(1-\phi)(y(t)-y(0))}{\lambda}\right)(\bmod 1)-S\right\}
$$

\footnotetext{
${ }^{10}$ This coincides with the social increasing returns condition high lighted in conventional multiple equilibria models (see Hammour [1989]).

${ }^{11}$ Note that, since the cross-section densities corresponding to different equilibria are rotations of one another, $\mathrm{d}_{R}\left(z_{t}, U\right)$ is constant across solutions, hence checking that $\mathrm{d}_{R}\left(z_{t}, U\right)>1 / k$ for one solution is enough to ensure uniqueness.

${ }^{12}$ This is the case, for example, if the density of $Y$ in $(13)$ is continuously differentiable and its first and second derivatives are integrable (see Caballero and Engel [1989a]).
} 
where $\phi=1 /(1+k)$.

Continuity: If the conditions ensuring existence hold for $0 \leq t \leq T,{ }^{13}$ there exists (at least) a set $\{r(t): 0 \leq t \leq T\}$ defining a sequence of equilibria (see (14)) such that the distribution of price deviations and output are continuous over this time period. Further, if $m(t)$ is differentiable and the conditions ensuring uniqueness also hold, then the (unique) output path is also differentiable.

Proof: Noting the equivalence between equations (3) and (4), all the statements on existence and uniqueness follow from the previous discussion. The continuity results follow from (15) and the fact that the assumptions made above ensure that $G_{t}(r)$ is differentiable (see Caballero and Engel [1989a]).

The conditions ensuring uniqueness imply that the right hand side of equation (15), as a function of $y$, defines a continuously differentiable contraction mapping. This has two interesting implications. First, since it is a contraction, standard fixed point calculations can be used to calculate in an efficient way the value of output at a given instant of time this is applied in the following section. Second, since it is continuously differentiable, the unique value of $r(t)$ defining a solution at time $t$ (see (14)) - and therefore the distribution of firm's price deviations- evolves smoothly over time. The dynamic behavior of the economy therefore is consistent with the fixed pricing rules assumption, in the sense that firms adjust their prices smoothly. Since the path of money (and therefore the distribution of price deviations) have no jumps, there cannot be a positive fraction of firms adjusting their price in an infinitesimal time period.

\subsection{Multiple Equilibria}

This section shows that more than one equilibrium may attain when $k>0$ and the economy is sufficiently far away from the steady state. The argument given in Section 4.1 shows that in this case the number of solutions of equation (10) is equal to the number of times the periodic function $G_{t}(r)$, with period $\lambda / k$, crosses the diagonal bisecting the square determined by $S-G_{\max }$ and $S-G_{\min }$ on the $x$-axis, and $G_{\min }$ and $G_{\max }$ on the $y$-axis; where $G_{\max }$ and $G_{\min }$ denote largest and smallest values $G_{t}(r)$ can take. It follows

\footnotetext{
${ }^{13}$ Recall that $m(t)$ is assumed to be continuous throughout this paper.
} 


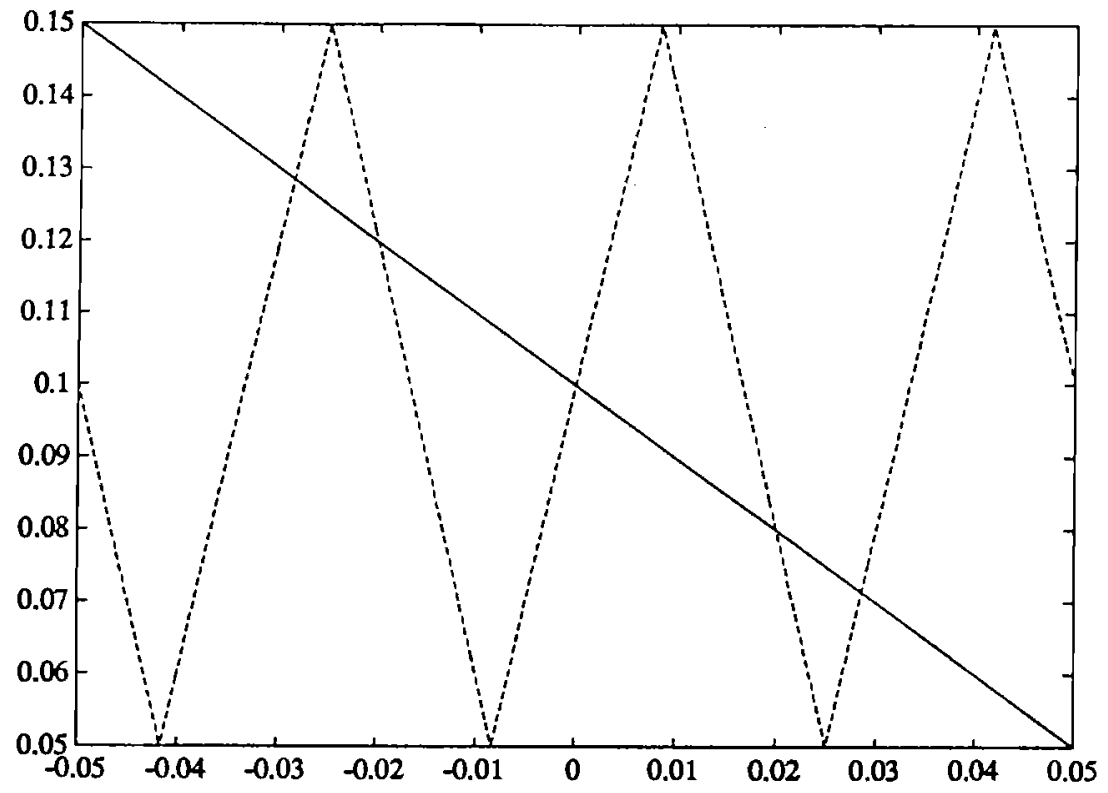

Figure 1: Example with more than one equilibrium

$$
L(r):(-) G_{t}(r):(\cdots)
$$

that the number of equilibria at time $t, N_{t}$, satisfies:

$$
N_{t} \geq \frac{2\left(G_{\max }-G_{\min }\right) k}{\lambda}-1
$$

Figure 1 shows $L(r)$ and $G_{t}(r)$ (for $t=0$ ) in a particular example, where there are no idiosyncratic shocks and the cross-section distribution of firms within their price cycle is uniform on $\left[\frac{1}{4}, \frac{3}{4}\right], \lambda=0.2, k=6$, and $m(0)=0$. For this example $G_{\max }=0.15$ and $G_{\min }=0.05$, and the number of equilibria is 5 .

It is shown in Appendix B that $G_{\max }-G_{\min }$ is equal to $\lambda \mathrm{d}_{I}\left(\mathrm{c}_{t}, U\right)$, where $\mathrm{d}_{I}\left(c_{t}, U\right)$ denotes a measure of the distance from the equilibrium distribution of firms' positions 
within their price cycle to the steady state. ${ }^{14}$ Equation (16) then leads to:

$$
N_{t} \geq 2 k \mathrm{~d}_{I}\left(c_{t}, U\right)-1
$$

In Section 6 we show that $\mathrm{d}_{I}\left(c_{t}, U\right)$ can also be interpreted as a measure of the effectiveness of monetary policy. The close relation between the distance from the steady state and the effectiveness of monetary policy is one of the themes explored in that section.

Usually the number of solutions of (10) is not significantly larger than the lower bound provided by (17). Equation (17) shows that, other things equal, the number of solutions for (10) grows at a rate approximately linear in (a) the degree of strategic complementarity, $k$; (b) the degree of monetary effectiveness and -equivalently to (b) - (c) the distance of the distribution of price deviations from its steady state.

The fact that equation (10) may have more than one solution does not necessarily imply that dynamic multiple equilibria are possible. The solutions of (10) give all possible distributions of price deviations an economy may have when the previous path of this distribution is disregarded. Yet the presence of menu-costs rules out jumps from one equilibrium to another. The economy's path prior to the time instant $t$ may uniquely determine the equilibrium it at tains at time $t$. Multiple equilibria that persist over time must take into account this dynamic consistency condition. This implies that a continuum of equilibria must exist at some instant in time. A general statement on this topic is an open research question.

\section{Output Fluctuaticns and Strategic Complementarity}

In this section we study the economy's aggregate out-of-steady-state behavior. We concentrate on the asymmetries introduced by strategic complementarity, and the desynchronizing features of idiosyncratic shocks. Even though many of the results extend to the more general setting described in Section $2,{ }^{15}$ for expository reasons we assume bandwidths and demand elasticities are equal across firms. We first consider the effect of strategic complementarity in isolation and assume there are no idiosyncratic shocks (Section 5.1).

\footnotetext{
${ }^{14}$ More precisely, $\mathrm{d}_{I}\left(c_{t}, U\right)$ is equal to the largest absolute error made when approximating the probability that $z_{t}$ belongs to any given interval (mod 1) by the probability the steady state distribution assigns to that event.

${ }^{15}$ See Caballero and Engel [1989b].
} 
Idiosyncratic shocks are incorporated in Section 5.2 .

\subsection{Strategic Complementarity and Fluctuations}

We begin with a simple but illustrative example that motivates the issues we formalize later. The economy is initially at the steady state described by Proposition 1 , when an increase in the rate of core money growth doubles firms' bandwidths. ${ }^{16}$ Bands are symmetric before and after the structural change. The cross-section distribution of firms within their pricing cycle, which was uniform on $[0,1)$ before the change, is uniform on $\left[\frac{1}{4}, \frac{3}{4}\right)$ after bands widen. ${ }^{17}$ Both before and after the change in core money growth, the money stock increases monotonically and continuously.

We first consider the case where there are no strategic interactions: firms' frictionless optimal prices increase one-for-one with increases in the money stock because substitution and income effects cancel. Firms' pricing decisions do not depend on the price level per se, but only on the money stock. Since there is a gap between the distribution of firms' positions within their pricing cycle and their trigger level, there is a period of time during which no firm reaches its trigger point and nominal prices remain unchanged. This period lasts until the (log of the) money stock grows by $\lambda / 4$. Real balances, and therefore output, increase at the same rate as the money stock during this period. By the time the first firm reaches its trigger level - this firm was about to increase its price when the structural change took place- firms begin changing their prices at a rate that is twice the steady state rate, and therefore output decreases at the same speed at which the money stock is growing. By the time the last firm completes its pricing cycle, the situation reverts again and output increases at the rate at which the money stock grows. In the absence of idiosyncratic shocks this cyclic behavior continues forever. The "curve" corresponding to $k=0$ in Figure 2 shows how output fluctuates when the rate of money growth is constant. If money grows at a stochastic rate, output increases at the same rate that the money stock until $m(t) \leq \lambda / 4$. Output then decreases - at the same rate that the money stock is growing- until $m(t)=5 \lambda / 4$, and so on. The frequency with which firms adjust prices

\footnotetext{
${ }^{10}$ This extreme example has the nice property: $l_{E}(0)-l_{C}(0)=0$ (see below), which allows us to isolate more clearly the effects arising from strategic complementarities from those arising from the shape of the cross sectional distribution.

${ }^{17}$ This ignores the effect of the expected rate of infiation on the demand for real balances. Once this effect is incorporated, the cross-section distribution of the $c_{i}$ 's continues being uniform on an interval of length $1 / 2$ (as long as this effect is not larger than $\lambda / 4$ ), but this interval is not centered around $1 / 2$. Except for a shift in the time axis, the analysis that follows remains valid.
} 
- equal to $m^{\prime}(t) / \lambda$ in the deterministic case- is not constant anymore, but on average it is equal to $\bar{m} / \lambda$, where $\bar{m}$ denotes the (new) average rate of money growth.

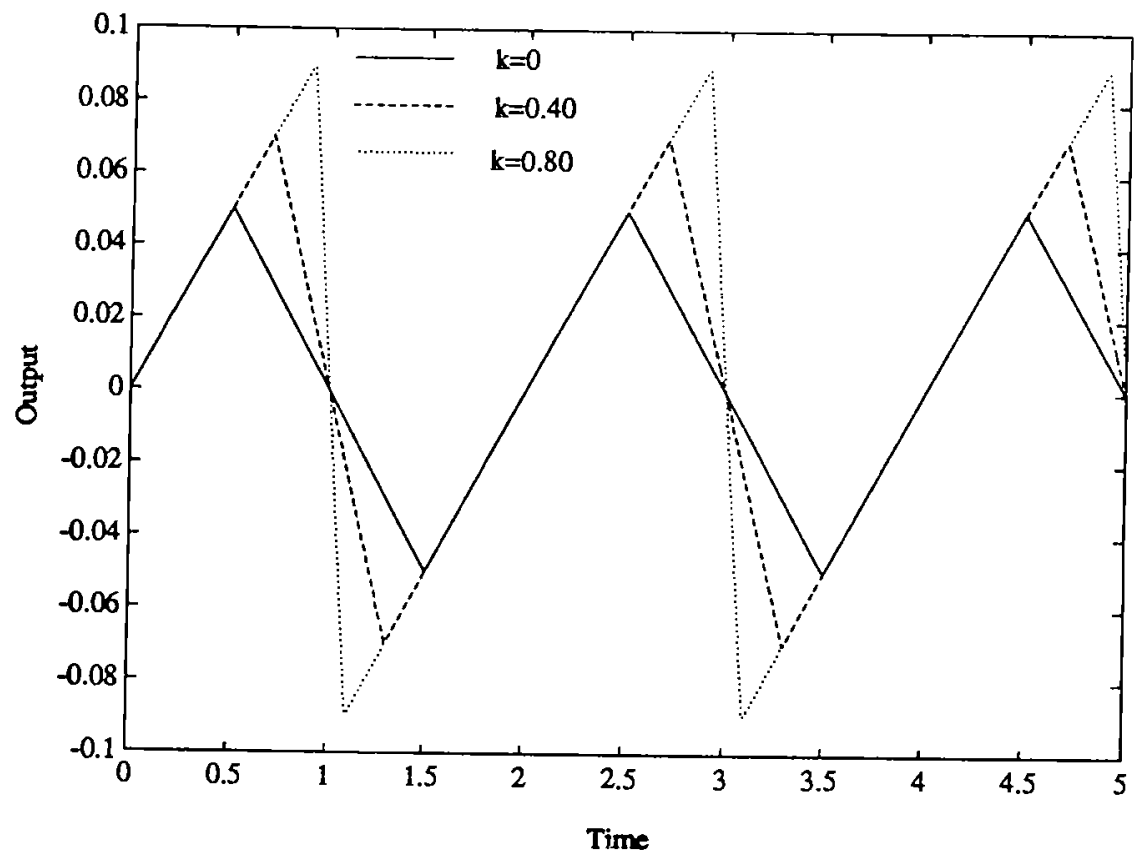

Figure 2: Output fluctuations with no idiosyncratic shocks

Next we consider the case with strategic interactions, to ensure a unique equilibrium we assume $k<1$. Firms' frictionless prices grow at a rate equal to a convex combination of the rates at which money and the price level are growing. A firm's frictionless price is less sensitive to increases in the money stock for larger values of the strategic complementarity parameter $k$. By the same token, the speed with which a firm moves in its pricing cycle becomes more sensitive to the changes in price level as $k$ becomes larger. Following the widening of bands, there is a period during which no firm adjusts its price, just as in the case with $k=0$. Since the price level remains constant, during this period, firms' frictionless prices grow slower than in the case without interactions. Yet the corresponding increase in real balances is amplified - by the presence of strategic interactions - into an increase in output that exactly offsets this effect, and output grows at the same speed as in the case 
$k=0$ for a longer period of time. By the time firms begin to adjust their prices, output falls sharply. The price level begins to increase (at a speed twice as large as the growth rate of money) and therefore firm's frictionless prices increase faster than they would if firms only considered changes in the money stock. Since the cross-section distribution of price deviations moves faster during the downturn, this period is shorter than it would be with $k=0$. When all firms have completed their first pricing cycle, output begins to increase and the cycle starts again.

Figure 2 shows output fluctuations when money grows at a constant rate, for various degrees of strategic complementarity. ${ }^{18}$ Two regularities emerge from this figure and the preceding discussion. First, output increases (decreases) when the rate at which firms are changing their prices is smaller (larger) than the corresponding steady state rate. Second, other things equal, output grows for a longer period of time - and declines for a shorter period of time - the larger the degree of strategic complementarity. These insights hold -in the absence of idiosyncratic shocks - for any distribution of firms within their cycle. Later in this section we show that:

$$
y^{\prime}(t)=\frac{1-f_{t}\left(1^{-}\right)}{1-(1-\phi) f_{t}\left(1^{-}\right)} m^{\prime}(t)
$$

where $f_{t}\left(1^{-}\right)$denotes the cross-section density of firms' positions within their pricing cycle at time $t$ and $\phi=1 /(1+k)$. Since the denominator is positive (this is required to ensure uniqueness), the numerator determines the sign of $y^{\prime}(t)$ and the strategic complementarity parameter, $k$, the magnitude of this rate of change.

The asymmetry described above implies that, other things equal, the average Iength of expansions is larger the larger the value of $k$. This is valid more generally than this example may suggest. Given an initial cross-section distribution of firms within their pricing cycle, $c_{0}$, and a degree of strategic complementarity $k$, let $l_{E}(k)$ denote the fraction of time output is growing and $l_{C}(k) \equiv 1-l_{E}(k)$. The difference between $l_{E}(k)$ and $l_{C}(k)$ measures the degree of asymmetry in the lengths of expansions and contractions. Assume there are no idiasyncratic shocks and the money stock grows at a constant rate - the expression that follows holds (approximately) in expectation when the stochastic process generating the

\footnotetext{
${ }^{18}$ Bandwidths are equal to 0.2 and $m(t)=0.01 t$ in this figure.
} 
money stock grows at a rate that is independent from the current level of output. Then:

$$
l_{E}(k)-l_{C}(k)=l_{E}(0)-l_{C}(0)+k \mathrm{~d}_{V}\left(\mathrm{c}_{t}, U\right),
$$

where $\mathrm{d} v\left(c_{t}, U\right)$ denotes a measure of distance - known as the variation distance - between $c_{t}$ and the steady state uniform distribution $U .{ }^{19}$ This result is proved in Lemma B10 in Appendix B. It shows that the asymmetry in fluctuations grows (linearly) both with the economy's distance from the steady state and the degree of strategic complementarity. ${ }^{20}$

When the economy is expanding, most firms are at the beginning of their pricing cycle and, other things equal, the larger the degree of strategic complementarity, the larger the incentive firms have not to adjust their prices. Hence expansions are reinforced by the presence of complementarities among firms, and their duration grows with $k$. Similarly, contractions are associated with periods where firms change their prices at a rate faster than average. When prices are strategic complements, the larger the number of firms that change their price, the larger the incentives other firms have to do the same. It follows that contractions are shorter the larger the degree of strategic complementarity.

The magnitude of the effects described above is proportional to the difference between the rate at which firms are adjusting their prices and the corresponding steady state rate, and this is proportional to the distance of the distribution of price deviations from the steady state. This explains why the asymmetry between the lengths of expansions and contractions increases with the distance from the steady state.

\subsection{Idiosyncratic Shocks and Fluctuations}

When the economy is forced away from the steady state described in Proposition 1, idiosyncratic shocks (whose increments do not depend on firms' current prices) bring the distribution of price deviations closer to its steady state and therefore dampen output fluctuations. The discussion of this mechanism is given in Caballero and Engel [1991]; and extended here to incorporate the presence of strategic interactions. If shocks are nonstationary, and no structural change takes place, the cross-section distribution of firms' positions within

\footnotetext{
${ }^{19}$ The variation distance belwcen $c_{\ell}$ and $U$ is equal to $\sup _{A}\left|\operatorname{Pr}\left\{c_{\ell} \in A\right\}-\operatorname{Pr}\{U \in A\}\right|$, where the supremum is taken over all Borel sets $A$. Note that, since there are no idiosyncratic shocks, $\mathrm{d} v\left(c_{\ell}, U\right)$ remains constant over time.

${ }^{20}$ This qualitative nature of this result may be expected to extend to the case where adjustment rules are two-sided, as long as core-inflation is positive.
} 
their pricing cycle converges to the uniform distribution. ${ }^{21}$ As time passes, the economy resembles more and more the steady state description given in Proposition 1.

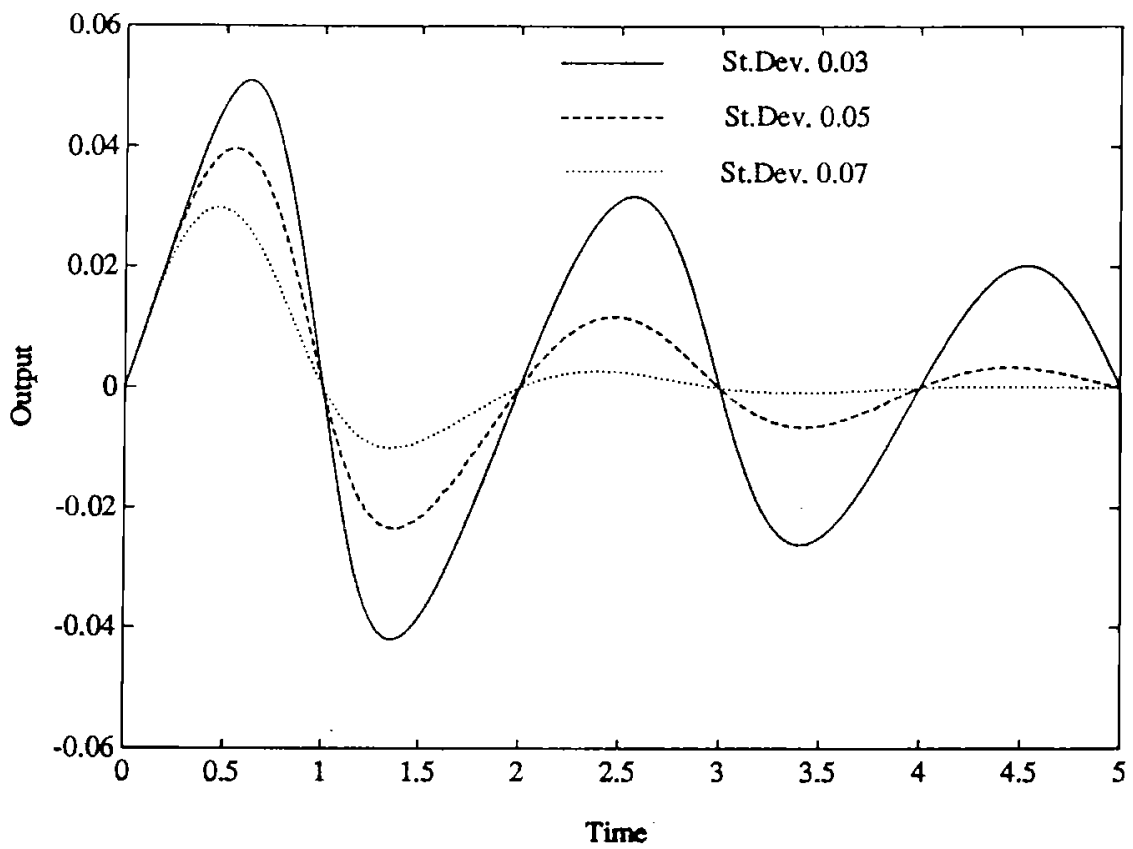

Figure 3: Output fluctuations and idiosyncratic shocks

Let us consider again the example where an increase in core money growth leads to a doubling of firms' bandwidths. We assume idiosyncratic shocks are normally distributed with zero mean and variance growing linearly with time. ${ }^{22}$ Figure 3 shows how output fluctuates on its way to the steady-state in the presence of idiosyncratic shocks for three different values of the variance, where time is measured in years. ${ }^{23}$ It is apparent form this figure that fluctuations dampen out faster the larger the (instantaneous) variance of firm specific shocks. Since shocks are non stationary, their desynchronizing effect increases

\footnotetext{
${ }^{21}$ Given the form of the distribution of price deviations derived in Section 4, the corresponding proofs follow directly from Caballero and Engel [1991].

${ }^{22}$ Strictly speaking, we should use a truncated normal (in any interval of length $d t$ ) with truncation point at $-d m(t)$. This is of second order importance when $m^{\prime}(t)>\sigma^{2}$. This approximation is also used in Proposition 3 below.

${ }^{23}$ These figures assume $k=0.40, \lambda=0.20$ and $m(t)=0.1 t$.
} 
without bound, hence output converges to its steady state level and the distribution of firms within their cycle approaches the steady state distribution. The larger the variance of idiosyncratic shocks, the faster the economy approaches its steady state distribution.

Figure 4 shows the path of output for three different degrees of strategic complementarity - and the same variance of idiosyncratic shocks. Figure 4 can be interpreted as the figure that results from adding idiosyncratic shocks (with instantaneous standard deviation equal to 0.05) to every one of the output paths considered in Figure 2. It is apparent that the asymmetry between the lengths of expansions and contractions persists in the presence of idiosyncratic shocks - during the time period where the economy is sufficiently far away from its steady state. This is consistent with equation (19). In the following proposition we extend equation (18) to the case where idiosyncratic shocks are also present.

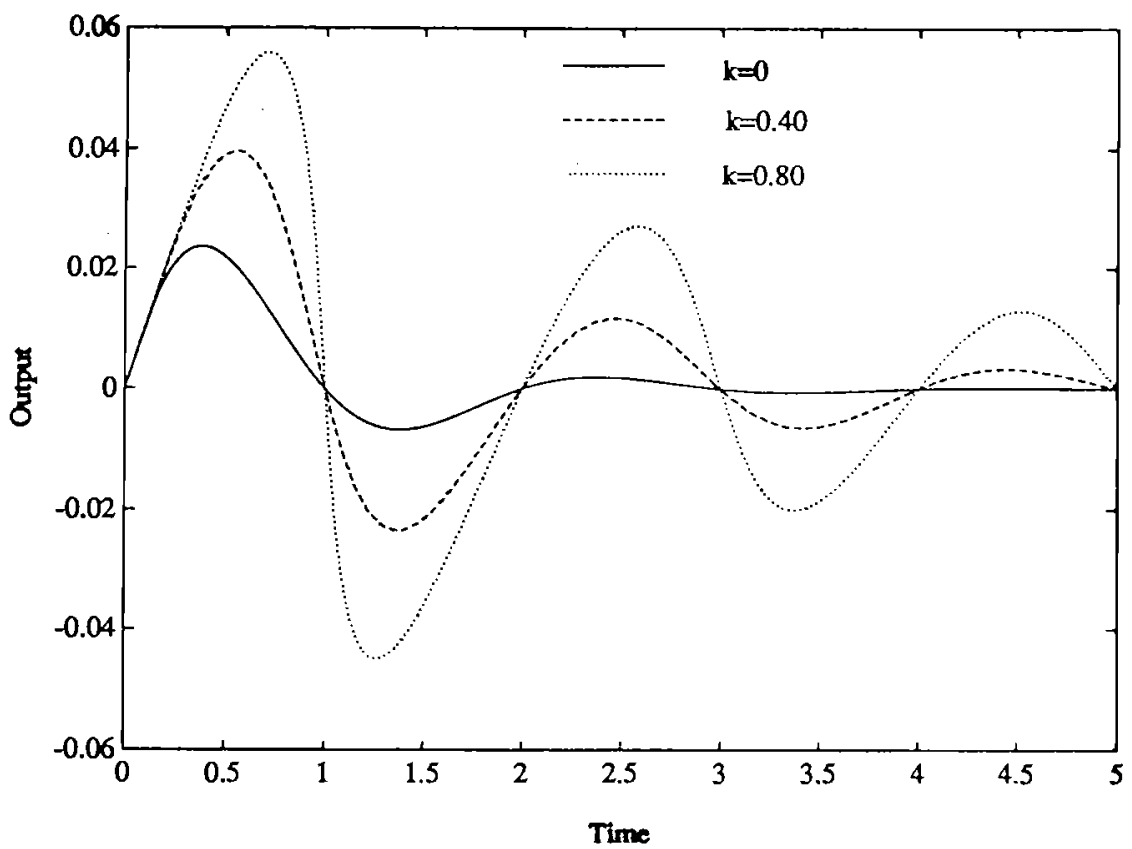

Figure 4: Output fluctuations, idiosyncratic shocks and strategic complementarity

Proposition 3 Suppose the cross-section distribution of idiosyncratic shocks - the $w_{i}(t)$ 's in equation (3) - is normal with zero mean and variance $\phi^{2} \sigma^{2} t$, with $\phi=1 /(1+k)$, and 
let $f_{t}(c)$ denote the density of $c_{t} \cdot{ }^{24}$

Then:

$$
y^{\prime}(t)=\frac{\left(1-f_{t}\left(1^{-}\right)\right) m^{\prime}(t)-x^{2} f_{t}^{\prime}\left(1^{-}\right)}{1-(1-\phi) f_{t}\left(1^{-}\right)}
$$

where $f_{t}^{\prime}(c)$ denotes the derivative (with respect to $c$ ) of $f_{t}(c)$, and $x^{2}=\phi^{2} \sigma^{2} / 2 \lambda$.

Proof: See Lemma B9 in Appendix B.

Equation (20) corresponds to (18) with an additional term in the numerator. This additional term takes into account the fact that now firms are moving within their pricing cycle not only because of increases in the money stock, but also because of firm specific shocks. As the economy approaches its steady state, both $\left(1-f_{t}\left(1^{-}\right)\right)$and $f^{\prime}{ }_{t}\left(1^{-}\right)$are tending to their steady state values (zero). Since the monotonicity assumption implies that the aggregate drift must be larger than the standard deviation of instantaneous shocks, the first term in the numerator of (20) dominates over the second term, and the discussion from Section 5.1 extends to the case with idiosyncratic shocks.

Summing up, when an $(S, s)$ economy with idiosyncratic shocks is forced away from the steady state described in Proposition 1, output oscillates on its way back to the steady state. Expansions are flatter and contractions are more pronounced (but shorter lived), the larger the degree of complementarity between firms' pricing decisions and the further away the economy is from its steady state.

\section{Average neutrality}

The previous section showed that monetary policy is generally not neutral when the economy is outside its steady state. Yet knowledge of the level of output (i.e. of $-(1+$ k) $\int_{0}^{1} z_{i} d i$ ) over some period of time (so as to know its derivative) is necessary to take advantage of non-neutrality. In this section it is shown that money is neutral on average when there is no information on the location of the distribution of price deviations. This serves as a threshold since any amount of information breaks the average neutrality result. ${ }^{25}$ We also show that the potential magnitude of the effect of monetary policy grows with the distance of the economy from its steady state. This ties in the various notions of distance

\footnotetext{
${ }^{21}$ It follows from the model derived in Appendix $A$ that $w_{t}=\phi v_{t}$, where the $v_{t}$ correspond to a linear combination of the (logs of the) actual shocks; see Section 2 and Appendix A. The assumption made above is therefore equivalent to $v_{t}$ having variance $\sigma^{2} t$.

${ }^{25}$ This paper does not deal with agents' responses to systematic exploitation of information. See Caballero and Engel [1989a] for preliminary discussion of monetary policy in the context of $(S, s)$ economies.
} 
that appeared in the preceding sections. For simplicity we assume that bandwidths and demand elasticities are the same across firms, ${ }^{26}$ and that the cross-section distribution of idiosyncratic shocks is normal (with a variance that increases with time).

The elasticity of output with respect to (continuous) money changes is introduced in order to make precise the concept of money neutrality. It also is useful when defining various measures of the potential magnitude of monetary policy effects. Its value at time $t$ is equal to:

$$
I_{t}=\frac{M}{Y} \frac{d Y}{d M}(M)=\frac{d y(m(t), t)}{d m},
$$

where $y(m, t)$ denotes output as a function of the current money stock, $m$, and the distribution of idiosyncratic shocks accumulated until time $t$. This index is shortsighted because it only reflects the effect of money on the current level of activity. ${ }^{27}$ It also assumes that the increase in the money stock does not affect the average growth rate of money, hence firms' inaction range. It measures the effect of output of a infinitesimal. continuous increase in the money stock.

From equation (5) it follows that $I_{t}$ is equal to minus $(1+k)$ times the derivative of $\int z_{i} d i$ with respect to the (logarithm of the) money stock at that instant in time. Hence, if on average prices have been changed recently, an increase in the money stock raises real balances and total output (i.e. lowers $\int_{0}^{1} z_{i} d i$ ): $I_{t}>0$. Conversely, an increase in the money stock is likely to reduce output if on average prices have not been changed for a long time: $I_{t}<0$.

Defining neutrality at time $t$ as having $I_{t}$ equal to zero is too general a definition. An infinitesimal increase in the money stock has no effect on activity every time output reaches a locally extreme (maximum or minimum) value and therefore $I_{t}$ is equal to zero at these instants in time. ${ }^{28}$ Yet the index of (instantaneous, myopic) monetary effectiveness, $I_{i}$, is different from zero an instant of time later. One measure for the distance of the economy from its steady state at time $t$ is the largest value $I_{\Delta}$ could take over all possible realizations of the (continuous and increasing) output path $m(s) ; s \geq t$. Let therefore:

$$
M_{1}(t)=\sup _{m(s) \geq m(t), s \geq t} I_{t}(m(s), s) .
$$

\footnotetext{
${ }^{26}$ The case with different bandwidths was considered in a previous version of this paper.

${ }^{27}$ Generating a boom today comes at the cost of a recession, usually milder than the boom, in the future. Intertemporal tradeoffs issues like this one are addressed in Caballero and Engel [1989a].

${ }^{28}$ When the path of output is continuous.
} 
It is shown in Lemma B11 in Appendix B that $M_{1}(t)$ is equal to $(1+k)$ times the largest relative error made when approximating the cross-section distribution of firms within their pricing cycle, $c_{t}$, by its steady state distribution. The condition ensuring uniqueness derived in Section 4 may now be interpreted in the following way. Once the (instantaneous) effect of monetary policy, as measured by $M_{1}(t)$, is smaller than $(1+k) / k$, there exists a unique equilibrium.

Next we study to what extent an uninformed policy maker can take advantage of the non-neutrality of money when the economy is away from its steady state. Assume that the order in which firms change their prices is known, yet the exact position of any firm within its pricing cycle is not known. This is equivalent to knowing the distribution of firms positions within their $(S, s)$ bands, except for a location parameter, $\psi$, that may take values between 0 and $\lambda$. The effectiveness of monetary policy depends on the actual value of $\psi$. Denote the corresponding money-elasticity of output by $I_{t}(\psi)$. Then (see Lemma $B 12$ in Appendix B)

$$
\int_{0}^{\lambda} I_{t}(\psi) d \psi=0
$$

This means that if the policy maker assigns equal probability to all possible locations of the distribution of firms' positions within their $(S, s)$ band, then monetary policy is neutral on average. The magnitude of (infinitesimal) monetary shocks may be expected to be larger the further away from the steady state the economy is. A measure of the average magnitude of monetary shocks (at time $t$ ), when the policy maker has no knowledge about the location of the distribution of price deviations, is given by:

$$
M_{2}(t)=\int_{0}^{\lambda}\left|I_{t}(\psi)\right| d \psi
$$

It is shown in Lemma $\mathrm{B} 13$ in Appendix $\mathrm{B}$ that $M_{2}(t)$ is equal to $(2 \lambda / \phi)$ times $\mathrm{d}_{V}\left(c_{t}, U\right)$, where $\mathrm{d} v\left(c_{t}, U\right)$ denotes the largest error made when approximating probabilities of events under $z_{t}$ by the corresponding probability under the steady state distribution. This is the notion of distance - known as the variation distance- related to the asymmetry between the lengths of expansions and contractions in Section 5.1. This asymmetry therefore grows with the size of the potential effects of (infinitesimal) money shocks.

An alternative measure of monetary policy effectiveness at time $t$ is the difference between the largest and smallest values output can take - from time $t$ onwards - over all possible continuous, increasing paths of $m(t)$. This leads to the following index of mone- 
tary policy effectiveness:

$$
M_{3}(t)=\sup _{m(s) \geq m(t)} y(m(s), s)-\inf _{m(s) \geq m(t)} y(m(s), s)
$$

The index $M_{3}(t)$ is equal to $(1+k)$ times the largest error made when approximating the probability of intervals $(\bmod 1)$ under $c_{t}$ by the corresponding probability under the steady state distribution (see Lemma B7 in Appendix B). The corresponding concept of distance between random variables is known as "discrepancy" - it is proportional to the (lower bound) for the number of equilibria derived in Section 4 .

Average neutrality holds independently of how far from the steady state the economy might be. Increasing money without worrying about the current output level has no average effect on output. The difference with full neutrality is that $M_{1}(t), M_{2}(t)$ and $M_{3}(t)$-and typically $I_{t^{-}}$are different from zero when the economy is not at its steady state. Increases in the money stock raise output during recessions but lower it during booms in such a way that these effects cancel each other. It may appear that this contradicts the result we derived in the preceding section, according to which booms are longer -and recessions shorter - the larger the degree of strategic interactions. Yet, as shown in Proposition 3 , the speed with which output grows during expansions is decreasing in the degree of strategic complementarity. Similarly, the larger the value of $k$, the faster output falls during contractions. These effects exactly cancel off the asymmetry between the lengths of booms and recessions so that monetary policy is neutral on average. An absolutely uninformed monetary a uthority cannot exploit (on average) situations where $M_{1}(t), M_{2}(t)$ or $M_{3}(t)$ are greater than zero.

\section{CONCLUSION}

This paper begins by generalizing Caplin and Spulber's [1987] steady state-money neutrality result, by allowing for strategic interactions and various sources of heterogeneity across firms. We then proceed to study non-steady state dynamics, first showing that whether a unique equilibrium can be guaranteed or not depends not only on the degree of strategic complementarity but also on how close the distribution of firms' positions in their price cycle is from the steady state. Next, we argue that strategic complementarities introduce realistic asymmetries into the business cycle; the stronger these complementarities are, the longer and smoother are expansions relative to contractions. Finally we demonstrate 
that the conditional correlation between money and output is typically non-zero outside the steady state, however the unconditional correlation remains zero. In other words, the steady state neutrality result no longer holds for every time $t$ but it holds on average.

Throughout the paper we assume that the band-policy remains invariant to the experiments we perform, and concentrate on distributional issues. For the most part, allowing for different bandwidths for different parameters values is unlikely to change the qualitative features of the results. ${ }^{29}$ This is not necessarily true, however, when we study the out-ofsteady-state behavior of an economy with strategic complementarities, since in this case the first best policy is likely to involve endogenous changes in firms' bands (i.e. fluctuating bands). In this sense, our results should be viewed as a first step towards understanding the complexities of stochastic dynamic menu cost economies with heterogeneous agents that are strategically related.

\footnotetext{
${ }^{29}$ As long as the value functions satisfy standard regularity conditions.
} 


\section{APPENDIX A}

This appendix briefly presents the basic model underlying the macroeconomic framework used in the paper. ${ }^{30}$ To shorten the formulae, the derivation from first principles of the demand side of the model is omitted.

There is a continuum of sectors indexed by the subscript $i \in[0,1]$, and within every sector a continuum of firms indexed by the subscript $j \in[0,1]$. Each sector faces at each time $t$ the following isoelastic demand function:

$$
Y_{i}^{d}(t)=\left(\frac{q_{i}(t)}{Q(t)}\right)^{-\theta} Y(t) \epsilon_{i}(t)
$$

with $Y_{i}^{d}(t)$ the quantity of the (composite) good $i$ demanded by consumers, $q_{i}(t)$ the price of the (composite) good $i, Q(t)$ the aggregate price index, $Y(t)$ aggregate expenditure, $\epsilon_{i}(t)$ the idiosyncratic shock to the demand for goods of sector $i$, and $\theta$ the price elasticity of the demand for good $i$.

Aggregate expenditure (equal to aggregate production in this model) is proportional to real balances:

$$
Y(t)=\frac{M(t)}{Q(t)}
$$

with $M$ denoting some measure of money holdings. Sectoral demands, as a function of relative prices, real balances, and idiosyncratic (sectoral) shocks, are obtained by replacing (23) in (22):

$$
Y_{i}^{d}(t)=\left(\frac{q_{i}(t)}{Q(t)}\right)^{-\theta}\left(\frac{M(t)}{Q(t)}\right) \epsilon_{i}(t) .
$$

Firm $j$ in sector $i$ faces a demand, $Y_{i j}^{d}(t)$, that depends on its relative price (within the sector ), $q_{i j}(t) / q_{i}(t)$, and on the total demand for the sector's composite good:

$$
Y_{i j}^{d}(t)=\left(\frac{q_{i j}(t)}{q_{i}(t)}\right)^{-\eta} Y_{i}^{d}(t),
$$

where $\eta>1$ is the price elasticity of the demand faced by firm $j$ in sector $i$. In this context

${ }^{30}$ This is a modified version of Blanchard and Kiyotaki's [1987] (henceforth BK) model. One difference with $B K$, and other similar models, is that consumers are assumed to solve a two stage-CES-budgeting problem within each period. They first decide how much to spend in each sector. Then they decide how to allocate these expenditures within each sector. Firms do not collude, and hence do not exploit the monopolistic structure of the first stage. This modification expands the parameter apace (demand elasticities) for which the results can be applied (an alternative way to achieve this is by changing the elasticity of output with respect to real balances). 
the frictionless price of a firm with costs of producing $Y_{i j}(t)$ equal to $\mathrm{c} Y_{i j}^{\alpha}(t) e_{i}(t)$, where $c$ is a constant, $\alpha$ a parameter greater than one that reflects increasing marginal costs and $e_{i}(t)$ a cost shock that affects all firms in sector $i$ equally, is:

$$
\frac{q_{i j}^{*}(t)}{Q(t)}=\left[A\left(\frac{q_{i}(t)}{Q(t)}\right)^{\eta}\left(\frac{q_{i j}(t)}{Q(t)}\right)^{-\eta}\right]^{\alpha-1} Y_{i}^{d}(t)^{\alpha-1} e_{i}(t)
$$

where $A=\left(\frac{\eta-1}{c \alpha \eta}\right)^{\frac{1}{1-\alpha}}$. All firms in sector $i$ are affected by the same shocks, have the same technology, and face identical demands. Therefore their equilibrium prices must be the same. Furthermore, if $q_{i j}(t)=q_{i j^{\prime}}(t)$ for all $\left(j, j^{\prime}\right) \in[0,1]$, then this must be the value of the sectoral price index, $q_{i}(t)$. Replacing this equilibrium result in (27) eliminates the subindex $j$ from now on:

$$
\frac{q_{i}^{*}(t)}{Q(t)}=A^{\alpha-1} Y_{i}^{d}(t)^{\alpha-1} e_{i}(t) .
$$

Equation (24) provides an expression for $Y_{i}^{d}(t)$. Substituting this in (27) yields:

$$
\frac{q_{i}^{*}(t)}{Q(t)}=\left[A\left(\frac{M(t)}{Q(t)}\right) V_{i}(t)\right]^{\phi}
$$

with $\phi \equiv(\alpha-1) /(1+\theta(\alpha-1))>0$ and $V_{i}(t) \equiv \epsilon_{i}(t) e_{i}(t)^{1 /(\alpha-1)}$. Without loss of generality it is assumed that $M(0)=Q(0)$ and $A=1$.

Working with the logarithms of the variables makes the algebra clearer. Therefore the following notation is introduced: $v_{i}(t) \equiv \log V_{i}(t), m(t) \equiv \log M(t), p_{i}^{*}(t) \equiv \log q_{i}^{*}(t)$, $p_{i}(t) \equiv \log q_{i}(t), P(t) \equiv \log Q(t), r_{i}(t) \equiv p_{i}(t)-P(t)$ and $r_{i}^{*} \equiv p_{i}^{*}(t)-P(t)$, where $p_{i}^{*}$ denotes the (frictionless) optimal price. A simple Cobb-Douglas aggregate price index (i.e. fixed weights) is adopted: $P(t) \equiv \int_{0}^{1} p_{i}(t) d i .^{31}$

When a "menu cost" is introduced, firms follow some sort of $(S, s)$ rule. The case of fixed $(S, s)$ bands, which is an approximation to the optimal pricing rule, is considered here. They can be proved to be first best only in very special cases in the context of our model. However, finding the true rule is technically very difficult and we have not yet found a solution (we suspect that firms haven't either). Nonetheless, very interesting results can be derived without questioning the optimality of the proposed $(S, s)$ rule too much.

An important role is played by the difference of the (log of the) actual price charged by sector $i$ and the (log of the corresponding) frictionless optimal price. The variable $z_{i}(t)$ is

\footnotetext{
${ }^{31}$ This index should be interpreted as an approximation of the more appropriate CES-index.
} 
defined as $z_{i}(t) \equiv p_{i}(t)-p_{i}^{*}(t), z_{i}(t) \in(s, S]$.

Simple algebra yields:

$$
p_{i}(t)=\phi(m(t)-P(t))+\phi v_{i}(t)+P(t)+z_{i}(t)
$$

Integrating with respect to $i$ on both sides of (29), imposing that (since shocks are idiosyncratic) $\int_{0}^{1} v_{i}(t) d i=0$, and using the definition of the aggregate price index, yields:

$$
P(t)=m(t)+\frac{1}{\phi} \int_{0}^{1} z_{i}(t) d i
$$

where $\int_{0}^{1} z_{i}(t) d i$ denotes the average percentage departure of the actual price of each sector with respect to its optimal price at time $t$. From this it follows that:

$$
p_{i}^{*}(t)=m(t)+\phi v_{i}(t)+\left(\frac{1-\phi}{\phi}\right) \int_{0}^{1} z_{i}(t) d i
$$

and

$$
-z_{i}(t)+S=S-\left(p_{i}(t)-p_{i}(0)\right)+\left(p_{i}^{*}(t)-p_{i}(0)\right)
$$

Let $\lambda=S-s$ denote the width of the range of percentage deviations of actual prices from their frictionless optimum. Taking $(\bmod \lambda)$ on both sides, and using the fact that $-z_{i}(t)+S$ belongs to $[0, \lambda)$ and $p_{i}(t)-p_{i}(0)$ is a multiple of $\lambda$, yields:

$$
z_{i}(t)=S-\left(S+p_{i}^{*}(t)-p_{i}(0)\right)(\bmod \lambda)
$$

It is easy to see that $z_{i}(t) \in(s, S]$ since due to the properties of the modulus operator, the second term on the right hand side of $(32)$ belongs to $[0, \lambda)$.

Finally, substituting (31) into (32) and denoting $k=(1-\phi) / \phi$ yields the fundamental equation of this paper:

$$
z_{i}(t)=S-\left(S+m(t)+\phi v_{i}(t)+k \int_{0}^{1} z_{u}(t) d u-p_{i}(0)\right)(\bmod \lambda)
$$

If we interpret $m(t)$ and $v_{i}(t)$ as deviations from their values at time $t=0$, and let $\Delta z_{i}(t) \equiv$ $z_{i}(t)-z_{i}(0)$, equation $(33)$ is equivalent to

$$
z_{i}(t)=S-\left(S+m(t)+\phi v_{i}(t)+k \int_{0}^{1} \Delta z_{u}(t) d u-z_{i}(0)\right)(\bmod \lambda)
$$


Equations (3) and (4) are then obtained by setting $w_{i}(t)=\phi v_{i}(t)$.

Substituting $Y(t)^{\beta_{i}}$ for $Y(t)$ in (22), and tracing the steps of the derivation leading to (33) and (34), yields analogous expressions for $z_{i}(t)$, with $k+1-\beta_{i}$ in the place of $k$.

\section{APPENDIX B}

LEMMA $\mathrm{B} 1$ Let $U$ denote a random variable uniform on $[0,1], X$ any random variable independent from $U$, and $Y \equiv(X+U)(\bmod 1)$. Then $Y$ is uniform on $[0,1]$.

Proof: Since $Y$ takes values in $[0,1]$, it suffices to show that its Fourier coefficients are equal to those of a distribution uniform on $[0,1]$. Thus it has to be shown that all non trivial Fourier coefficients of $Z$ are equal to zero. A calculation from first principles shows that, given any random variable $X$, the Fourier coefficients of $X$ and $X(\bmod 1)$ are the same. Hence the Fourier coefficients of $Y$ are equal to the product of those of $X$ and $U$. Since $U$ is uniform on $[0,1]$, its non trivial Fourier coefficients are equal to zero. It follows that all non trivial Fourier coefficients of $Y$ are also equal to zero, completing the proof.

LEMмA B2 Let $X$ be a random variable whose density $f(x)$ has bounded variation. Then $X(\bmod 1)$ also has a density, $f_{1}(x)$, and

$$
f_{1}(x)=\sum_{k} f(x+k)
$$

Now assume that the characteristic function of $X, \hat{f}(z)$, satisfies $\sum_{k \geq 1}|\hat{f}(2 \pi k)|<+\infty$. Then:

$$
f_{1}(u)=1+2 \sum_{k \geq 1} \Re\left[\hat{f}(2 \pi k) \mathrm{e}^{-i 2 \pi k u}\right],
$$

where $\Re[z]$ denotes the real part of the complex number $z$.

Proof: Equation (35) is is a well known result in probability theory, for a proof under the assumptions made above see Proposition 3.1 in Engel [1991].

Next we derive (36). Since the Fourier coefficients of $X$ and $X(\bmod 1)$ are the same, it follows that the Fourier coefficients of $X(\bmod 1)$ are summable and $X(\bmod 1)$ has a continuous density, $f_{1}(x)$, with bounded variation. Applying Poisson's Summation Formula (see Butzer and Nessel, 1971, p.202, for the version being used here) leads to the expression for $f_{1}(u)$. 
LEMMA B3 Let $X$ denote a random variable whose characteristic function $\hat{f}(z)$ satisfies $\sum_{k \geq 1}|\hat{f}(2 \pi k)|<+\infty$. Let $G(a)=\mathrm{E}[(X+a)(\bmod 1)]$. Then:

$$
\begin{aligned}
G(a) & =\frac{1}{2}-\frac{1}{\pi} \sum_{k \geq 1} \frac{1}{k} \Im[\hat{f}(2 \pi k)], \\
G^{\prime}(a) & =-2 \sum_{k \geq 1} \Re[\hat{f}(2 \pi k)],
\end{aligned}
$$

where $\Im[z]$ and $\Re[z]$ denote the imaginary and real parts of the complex number $z$, respectively, and $x(\bmod 1)$ the difference between $x$ and the largest integer less than or equal than $x$.

Proof: Substituting this expression for $f_{1}(x)$ derived in $(36)$ in $\mathrm{E}[X(\bmod 1)]=\int x f_{1}(x) d x$, interchanging the order of integration and summation, and integrating the resulting terms, leads to the expression for $G(a)$. Differentiating under the summation sign, ${ }^{32}$ leads to the expression for $G^{\prime}(a)$.

Lem ма B4 Assume $X$ satisfies the assumptions in Lemma B3, let $f_{1}(x)$ denote the density of $X(\bmod 1)$, and define $G(a)$ as in Lemma 53, for $0 \leq a \leq 1$. Then:

$$
G^{\prime}(a)=1-f_{1}(1-a)
$$

Proof: Follows directly from (38) and (36).

LEMMA B5 Let $P(A)$ denote a probability measure on $[0,1]$ that has density $f(x)$ with nespect to Lebesgue measure, and let $Q(A)$ denote Lebesgue measure on the unit interval. Define:

$$
\mathrm{d}_{R}(P, Q)=\sup _{A}\left|\frac{P(A)}{Q(A)}-1\right|
$$

where the supremum is taken over all sets $A$ in $[0,1]$ with positive Lebesgue measure. ${ }^{33}$ Then:

$$
\mathrm{d}_{R}(P, Q)=\sup _{x}|f(x)-1|
$$

Proof: Since $\lim _{d x \rightarrow 0} P([x, x+d x]) / d x=f(x)$, except for $x$ belonging to a set of Lebesgue measure zero (see Billingsley [1986, p.439]), letting $A=[x, x+d x]$ on the right hand side of $(39)$ shows that $\mathrm{d}_{R}(P, Q) \geq \sup _{x}|f(x)-1|$.

\footnotetext{
${ }^{32}$ Additional smoothness assumptions are required at this step, Caballero and Engel [1989a].

${ }^{33}$ Strictly speaking all the suprema mentioned in this lemma should be essential suprema.
} 
The remaining inequality needed to establish (40) follows from:

$$
\begin{aligned}
\left|\frac{P(A)}{Q(A)}-1\right| & =\left|\frac{\int_{A}(f(x)-1) d x}{Q(A)}\right| \\
& \leq \frac{\int_{A}|f(x)-1| d x}{Q(A)} \\
& \leq \frac{\int_{A} \sup _{x}|f(x)-1| d x}{Q(A)} \\
& =\sup _{x}|f(x)-1| .
\end{aligned}
$$

LEмMA B6 Let $X$ denote a random variable taking values in $[0,1]$ that satisfies the assumptions of Lemma B3, denote its density by $f_{1}(x)$, and define $G(a)$ as in Lemma B3. Let

$$
\mathrm{d}_{I}(X, U)=\sup _{A}|\operatorname{Pr}\{X \in A\}-m(A)|
$$

denote the notion of distance - from $X$ to a distribution $U$ uniform on $[0,1]-$ known as discrepancy, where the supremum is taken over all sets $A$ in $[0,1]$ that are either of the form $[a, b]$ or $[0, a] \cup[b, 1], 0 \leq a<b \leq 1$, and $m(A)$ denotes Lebesgue measure.

Then:

$$
\mathrm{d}_{I}(X, U)=\sup _{a} G(a)-\inf _{a} G(a)
$$

Proof: For $0 \leq a<b \leq 1$ in $[0,1]$ we have:

$$
G(b)-G(a)=\int_{a}^{b} G^{\prime}(u) d u
$$

Using Lemma B2 and a change of variables:

$$
\begin{aligned}
& =(d-c)-\int_{c}^{d} f_{1}(v) d v \\
& =m([c, d])-\operatorname{Pr}\{X \in[c, d]\}, \\
& =\operatorname{Pr}\{X \in[0, c] \cup[d, 1]\}-m([0, c] \cup[d, 1]),
\end{aligned}
$$

where $d=1-a$ and $c=1-b$. Equation (41) now follows from (42) and (43).

LеммA B7 With the notation of Section 6:

$$
M_{3}(t)=\sup _{m(s) \geq m(t)} y(m(s), s)-\inf _{m(s) \geq m(t)} y(m(s), s) .
$$

Proof: Let $X$ and $Y$ be independent random variables and denote $G_{X}(a)=\mathrm{E}[(X+$ 
$a)(\bmod 1)]$ and $G_{X+Y}(a)=\mathrm{E}[(X+Y+a)(\bmod 1)]$. Lemma $\mathrm{B} 6$ and the fact that idiosyncratic shocks are normal (with a variance that increases over time) imply that all that needs to be proved is that

$$
\sup _{a} G_{X+Y}(a) \leq \sup _{a} G_{X}(a)
$$

and

$$
\inf _{a} G_{X+Y}(a) \geq \inf _{a} G_{X}(a)
$$

Since both proofs are very similar, we only prove (44).

Conditioning on $Y=y$ and using the independence assumption yields:

$$
\begin{aligned}
G_{X+Y}(b) & =\int G_{X}(y+b) d F(y) \\
& \leq \int\left\{\sup _{a} G_{X}(a)\right\} d F(y) \\
& =\sup _{a} G_{X}(a) .
\end{aligned}
$$

Equation (44) now follows from the fact that the latter inequality holds for all $b$.

LEMMA B8 Given a random variable $X$, define:

$$
h(a, b)=\mathrm{E}\left[\left(X+Z_{b}+a\right)(\bmod 1)\right]
$$

where $Z_{b}$ denotes a random variable independent from $X$, normal, with zero mean and variance $b$. Let $f(y)$ denote the density of $Y \equiv\left(X+Z_{b}\right)(\bmod 1)$ and $f^{\prime}(y)$ its derivative. Then:

$$
\frac{\partial h}{\partial b}(a, b)=\frac{1}{2}\left(f^{\prime}(1-a)-1\right)
$$

Proor: Lemma B3 implies that:

$$
h(a, b)=\frac{1}{2}-\frac{1}{\pi} \sum_{k \geq 1} \frac{1}{k} \mathrm{e}^{-2 \pi^{2} k^{2} b} \Im\left[\hat{f}_{X}(2 \pi k) \mathrm{e}^{2 \pi k a}\right],
$$

where $\hat{f}_{X}(t)$ denotes the characteristic function of $X$. Straightforward calculations based upon (46) show that

$$
\frac{\partial h}{\partial b}(a, b)=\frac{1}{2} \frac{\partial^{2} h}{\partial a^{2}}(a, b)
$$

Substituting the expression that is obtained from Lemma B4 for $\partial h / \partial a$ in (47) completes the proof. 
Lemma B9 (Proof of Proposition 3)

Proof: Equation (15) in the main text is equivalent to:

$$
y(t)=\frac{\lambda}{\phi} H\left(\frac{m(t)-(1-\phi)(y(t)-y(0))}{\lambda}, \frac{x^{2} t}{\lambda}\right),
$$

where $H(a, b)$ is defined as in Lemma B8, with $c_{0}$ in place of $X$, and $x^{2}=\phi^{2} \sigma^{2} / 2 \lambda$. Implicitly differentiating both sides of (48) with respect to $t$, using Lemmas B4 and B8 to evaluate $\partial H / \partial a$ and $\partial H / \partial b$, and rearranging terms leads to (20) in the main text.

\section{Lemma B10 (Proof of Equation (19))}

Proof: From the definition of $l_{E}(k)$ it follows that:

$$
l_{E}(k)=\frac{1}{\lambda} \int_{0}^{\lambda} I\left\{m: \frac{\partial y}{\partial m}<0\right\} d m,
$$

with $I\{A\}$ denoting the indicator function of set $A$. An argument similar to that given in the Proof of Proposition 3 shows that:

$$
\frac{\partial y}{\partial m}=\frac{\left(1-f_{t}\left(1^{-}\right)\right)}{1-(1-\phi) f_{t}\left(1^{-}\right)}
$$

Since the denominator in (50) is positive, equation (49) is equivalent to:

$$
l_{E}(k)=\frac{1}{\lambda} \int_{0}^{\lambda} I\left\{m: f_{0}\left(\frac{m-(1-\phi)(y(m)-y(0))}{\lambda}\right)<1\right\} d m,
$$

with $f_{0}(c)$ denoting the density of $c_{0}$. Introducing the change of variable $v=m-(1-\phi) y(m)$ in (51) and using (50) to evaluate $d m$ in terms of $d v$ leads to:

$$
\begin{aligned}
l_{E}(k) & =\frac{1}{\lambda} \int_{0}^{\lambda} I\left\{v: f_{0}\left(\frac{v}{\lambda}\right)<1\right\}\left\{1+k\left(1-f_{0}\left(\frac{v}{\lambda}\right)\right)\right\} d v \\
& =l_{E}(0)+k \int_{0}^{1} I\left\{f_{0}(u)<1\right\}\left(1-f_{0}(u)\right) d u .
\end{aligned}
$$

The proof now follows from two elementary properties of the variation distance. First, $\mathrm{d} v\left(c_{0}, U\right)=\int_{0}^{1} I\left\{f_{0}(u)<1\right\}\left(1-f_{0}(u)\right) d u$. Second, since $c_{t}$ is obtained by rotating $c_{0}$, $\mathrm{d}_{V}\left(c_{t}, U\right)=\mathrm{d}_{V}\left(c_{0}, U\right)$. The identity above then implies that

$$
l_{E}(k)=l_{E}(0)+k \mathrm{~d}_{V}\left(c_{t}, U\right)
$$


LEMMA B11 With the notation introduced in Section 6 we have

$$
M_{1}(t)=(1+k) \mathrm{d}_{R}\left(c_{t}, U\right)
$$

Proof: See Section 4 in Caballero and Engel [1991].

LEMMA B12 With the notation of Section 6:

$$
\int_{0}^{\lambda} I_{t}(\psi) d \psi=0
$$

Proof: Let $G(a)=\mathrm{E}[(X+a)(\bmod 1)]$, with $X=c_{0}+\left(w_{t} / \lambda\right)$. Equations (14), (4) and (3) imply that $y(m, t)=-(1+k)\left(G(l)-\frac{1}{2}\right)$, with $l$ equal to a constant (that depends on $t$ ). It follows that $I_{t}(\psi)=G^{\prime}(l+\psi)$. Equation (52) now follows from this identity and the (trivial) fact that $G^{\prime}(a)$ is periodic, with period equal to one.

LEMMA B13 With the notation of Section 6 :

$$
\int_{0}^{\lambda}\left|I_{t}(\psi)\right| d \psi=\frac{2 \lambda}{\phi} \mathrm{d}_{V}\left(c_{t}, U\right)
$$

Proof: Using the same change of variables as in the proof of Lemma B10 leads to:

$$
\int_{0}^{\lambda}\left|I_{t}(\psi)\right| d \psi=\frac{\lambda}{\phi} \int_{0}^{1}\left|1-f_{t}(v)\right| d v
$$

where $f_{t}(v)$ denotes the density of $c_{t}$. Equation (53) now follows from an elementary property of the variation distance. 


\section{REFERENCES}

Akerlof, G. And J. Yellen: "Can Small Deviations from Rationality Make Significant Differences to Economic Equilibria?," American Economic Review 75 (1985), 708-721.

Ball, L. AND D. Romer: "The Equilibrium and Optimal Timing of Price Changes," NBER Working Paper 2412 (1987).

Barro, R.J.: "A Theory of Monopolistic Price Adjustment," Review of Economic Studies 39 (1972), 17-26.

Benabou, R.: "Optimal Price Dynamics and Speculation with a Storable Good," Econometrica 57-1 (1989), 41-81.

Billingsley, P.: Probability and Measure, $2^{\text {nd }}$ Ed. John Wiley, New York, 1986.

Blanchard, O.J. and N. KiYotaki: "Monopolistic Competition and the Effects of Aggregate Demand," American Economic Review 77-4 (1987), 647-666.

Butzer, P.L., AND R.J.Nessel: Fourier Analysis and Approximation Vol. 1 OneDimensional Theory, Academic Press, New York, 1971.

Caballero, R.J. and E.M.R.A. Engel: "The $S$-s Economy: Aggregation, Speed of Convergence and Monetary Policy Effectiveness," Columbia Univ. Working Paper \#420, (1989a).

Caballero, R.J. AND E.M.R.A. ENGEL: "Heterogeneity and Output Fluctuations in a Dynamic Menu Cost Economy," Columbia Univ. Working Paper \#453, (1989b).

Caballero, R.J. and E.M.R.A. Engel: "Dynamic $(S, s)$ Economies," Econometrica, (1991) (forthcoming).

CaPlin, A.S., AND D. Spulber: "Menu Costs and the Neutrality of Money," Quarterly Journal of Economics, 102-4 (1987), 703-726.

Cooper, R. AND A. John: "Coordinating Failures in Keynesian Models," Quarterly Journal of Economics 103 (1988), 441-464.

Engel, E.M.R.A.: A Road to Randomness in Physical Systems, Lecture Notes in Statistics, Springer Verlag, New York, 1991.

HaмmouR, M. "Indeterminacy and Instability in Macro Models with External Effects," (1989), Chapter II, MIT Ph.D. thesis. 
Mankiw, N.G.: "Small Menu Costs and Large Business Cycles: A Macroeconomic Model of Monopoly," Quarterly Journal of Economics 100- 2 (1985), 529-539.

Rotemberg, J.: "The New Keynesian Microeconomic Foundations," NBER Macroeconomics Annual (1987), 69-104.

Sheshinski, E., AND Y.Weiss: "Inflation and Costs of Price Adjustment," Review of Economic Studies, 44 (1977), 287-303.

Sheshinski, E., And Y.Weiss: "Optimum Pricing Policy under Stochastic Inflation," Review of Economic Studies, 50 (1983), 513-529. 